\title{
Quantification of structural cerebral abnormalities on MRI 18 months after aneurysmal subarachnoid hemorrhage in patients who received endovascular treatment
}

\author{
Jeroen de Bresser • Joanna D. Schaafsma • \\ Merel J. A. Luitse • Max A. Viergever • \\ Gabriel J. E. Rinkel • Geert Jan Biessels
}

Received: 15 September 2014 / Accepted: 20 November 2014 / Published online: 6 December 2014

(C) Springer-Verlag Berlin Heidelberg 2014

\begin{abstract}
Introduction Volume measurements performed on brain MRI after aneurysmal subarachnoid hemorrhage (aSAH) may provide insight into the structural abnormalities that underlie the commonly occurring and persistent long-term functional deficits after aSAH. We examined the pattern of long-term cerebral structural changes on MRI in relation to known risk factors for poor functional outcome.

Methods We studied MRI scans from 38 patients who received endovascular treatment and were not dependent for activities of daily life at 18 months after aSAH. Risk factors for poor functional outcome (clinical condition, Hijdra score, and bicaudate index on admission; occurrence of hydrocephalus or delayed cerebral infarction during hospitalization) were related to supratentorial cerebral parenchymal and lateral ventricular volumes on MRI with linear regression analyses adjusted for age, sex, and intracranial volume.

Results Clinical condition, Hijdra score, and bicaudate index on admission were not related to cerebral parenchymal volume at 18 months. A higher bicaudate index on admission was related to lateral ventricular enlargement at 18 months after aSAH (Beta; $95 \%$ CI: $0.51 ; 0.14 \leftrightarrow 0.88)$. Delayed cerebral infarction was related to smaller cerebral parenchymal volumes $(-0.14$;
\end{abstract}

J. de Bresser $(\bowtie)$

Department of Radiology, University Medical Center Utrecht,

P.O. Box 85500, Heidelberglaan 100, 3508 GA Utrecht,

The Netherlands

e-mail: j.debresser@umcutrecht.nl

J. D. Schaafsma • M. J. A. Luitse - G. J. E. Rinkel • G. J. Biessels Department of Neurology and Neurosurgery, Brain Center Rudolf Magnus, University Medical Center Utrecht, Utrecht,

The Netherlands

M. A. Viergever

Image Sciences Institute, University Medical Center Utrecht,

Utrecht, The Netherlands
$-0.25 \leftrightarrow-0.04)$ and to lateral ventricular enlargement (0.49; $0.16 \leftrightarrow 0.83$ ) at 18 months.

Conclusion Volume measurements of the brain are able to quantify patterns of long-term cerebral damage in relation to different risk factors after aSAH. Application of volumetric techniques may provide more insight into the heterogeneous underlying pathophysiological processes. After confirmation of these results in larger studies, volumetric measures might even be used as outcome measures in future treatment studies.

Keywords Brain segmentation · Brain MRI · Subarachnoid hemorrhage $\cdot$ Outcome $\cdot$ Cerebral abnormalities

\section{Introduction}

Aneurysmal subarachnoid hemorrhage (aSAH) results in a high case fatality and morbidity [1]. Even people who return home after aSAH without overt focal neurological deficits often have persistent cognitive deficits [2], and difficulty in resuming professional and social activities [3]. (Semi-)automated volume measurements performed on brain MRI may provide insight into the structural abnormalities after aSAH that underlie these functional deficits. However, application of volumetric techniques in patients after aSAH is challenging. Requirements of cerebral volume measurements for application on MRI scans after aSAH are a high accuracy and precision in the context of heterogeneous MRI abnormalities (e.g., infarcts, hemorrhage, cerebral damage due to ventricular drains and enlarged ventricles). Therefore, in patients after aSAH, these measurements have thus far been performed by only few studies (e.g., [4-6]). These studies showed cerebral structural changes compared to controls that correlated with functional outcome.

We have tuned a method for cerebral volume measurements specifically on a clinical MRI scanning protocol for 
use in patients after aSAH who received endovascular treatment; this resulted in a high accuracy [6]. In the present study, we apply this method to examine the pattern of long-term cerebral structural changes on MRI in relation to known risk factors for poor functional outcome after aSAH.

\section{Materials and methods}

\section{Participants}

Participants were retrieved from a prospectively collected cohort of consecutive patients who were independent for activities of daily life (modified Rankin Scale (mRS) $\leq 3$ [7]) after aSAH and who underwent digital subtraction angiography and MRA at the University Medical Center Utrecht to assess occlusion of aneurysms treated with coil placement at 18 months after aSAH [8]. For the present study, we included those patients who had been examined between May 2005 and September 2007. None of the 38 patients that were included had a past medical history of SAH, ischemic stroke, or hemorrhagic stroke. The study was approved by the medical ethics committee of the University Medical Center Utrecht, and all participants provided written informed consent.

\section{Clinical data}

Condition on admission was recorded at the time of the aSAH by the World Federation of Neurosurgical Societies SAH grading scale (WFNS) [9]. On all admission CT scans, the same rater quantified the amount of extravasated blood with the Hijdra score and the size of the ventricles with the bicaudate index [10,11]. The occurrence of symptomatic hydrocephalus (defined as decreased level of consciousness with increased bicaudate index (larger than the upper 95th percentile for age) [10]), delayed cerebral infarction (infarcts compatible with the clinical symptoms of delayed cerebral infarction (new focal deficits or decreased level of consciousness) after exclusion of other potential causes of clinical deterioration such as complications related to aneurysm treatment [12]) and rebleeding (defined as sudden deterioration of clinical condition and increased amount of extravasated blood on CT) during hospitalization were recorded. Clinical outcome was assessed at the time of the MRI scan by the mRS [7].

Cerebral MRI and image processing

All cerebral MRI scans were acquired on the same 3 T Philips magnetic resonance system with a standardized clinical MRI protocol $(24$ contiguous slices, voxel size: $0.45 \times 0.45 \times$ $\left.4.00 \mathrm{~mm}^{3}\right)$ and consisted of axial T1-weighted $(\mathrm{TR} / \mathrm{TE}=$ $500 / 10 \mathrm{~ms}$ ) and T2-weighted (TR/TE $=3000 / 80 \mathrm{~ms})$ images.
A method for probabilistic segmentation of cerebral structures [13] was tuned specifically to deal with the heterogeneous cerebral abnormalities seen in patients after aSAH [6]. The main adjustments consisted of implementation and tuning of the image processing algorithm in such a way that it could better deal with the large heterogeneity of lateral ventricular and peripheral cerebrospinal fluid volume and to minimize the effects that cerebral infarcts have on the measurements. This resulted in segmentations with a good accuracy and precision in patients after aSAH [6]. This method was performed on the T1and T2-weighted images to determine supratentorial cerebral parenchymal, peripheral cerebrospinal fluid, and lateral ventricular volumes. To date, no automated method can segment focal lesions with a high accuracy. Therefore, cerebral infarcts, ventricular drain trajectories, coil artifacts, and other less frequent brain abnormalities (e.g., meningioma's) were segmented manually. Supratentorial intracranial volumes were calculated by adding all other volumes (for details, see [6]). All segmentations were checked visually and considered to be accurate.

Statistical analysis

The relationships between risk factors for poor functional outcome after aSAH (clinical condition, Hijdra score, and bicaudate index on admission; symptomatic hydrocephalus and delayed cerebral infarction during hospitalization), and cerebral parenchymal volume and lateral ventricular volume 18 months after aSAH were examined by linear regression analyses adjusted for age, sex, and intracranial volume. Because of non-normal distribution (Kolmogorov-Smirnov, $p<0.05$ ), lateral ventricular volumes were natural-log-transformed for these analyses. In secondary analyses, we studied firstly the relationships between risk factors and presence of cerebral infarcts and infarct volumes in patients with infarcts and secondly, the relationship between cerebral parenchymal volume and lateral ventricular volume at 18 months after aSAH (relative to intracranial volume; dichotomized by a median split) and clinical outcome at 18 months after aSAH (dichotomized at a $\mathrm{mRS}<2$ or $\mathrm{mRS} \geq 2$ ) by logistic regression analyses adjusted for age and sex.

\section{Results}

Examples of the brain segmentations are shown for a participant without a cerebral infarct and a participant with a cerebral infarct (Figs. 1 and 2). Patient characteristics and cerebral volumes at 18 months after aSAH are shown in Table 1.

The relationships between different risk factors for poor functional outcome after aSAH and cerebral abnormalities 18 months after aSAH are shown in Table 2.

Measures of the initial impact of the hemorrhage (clinical condition, Hijdra score, and bicaudate index) were not related 

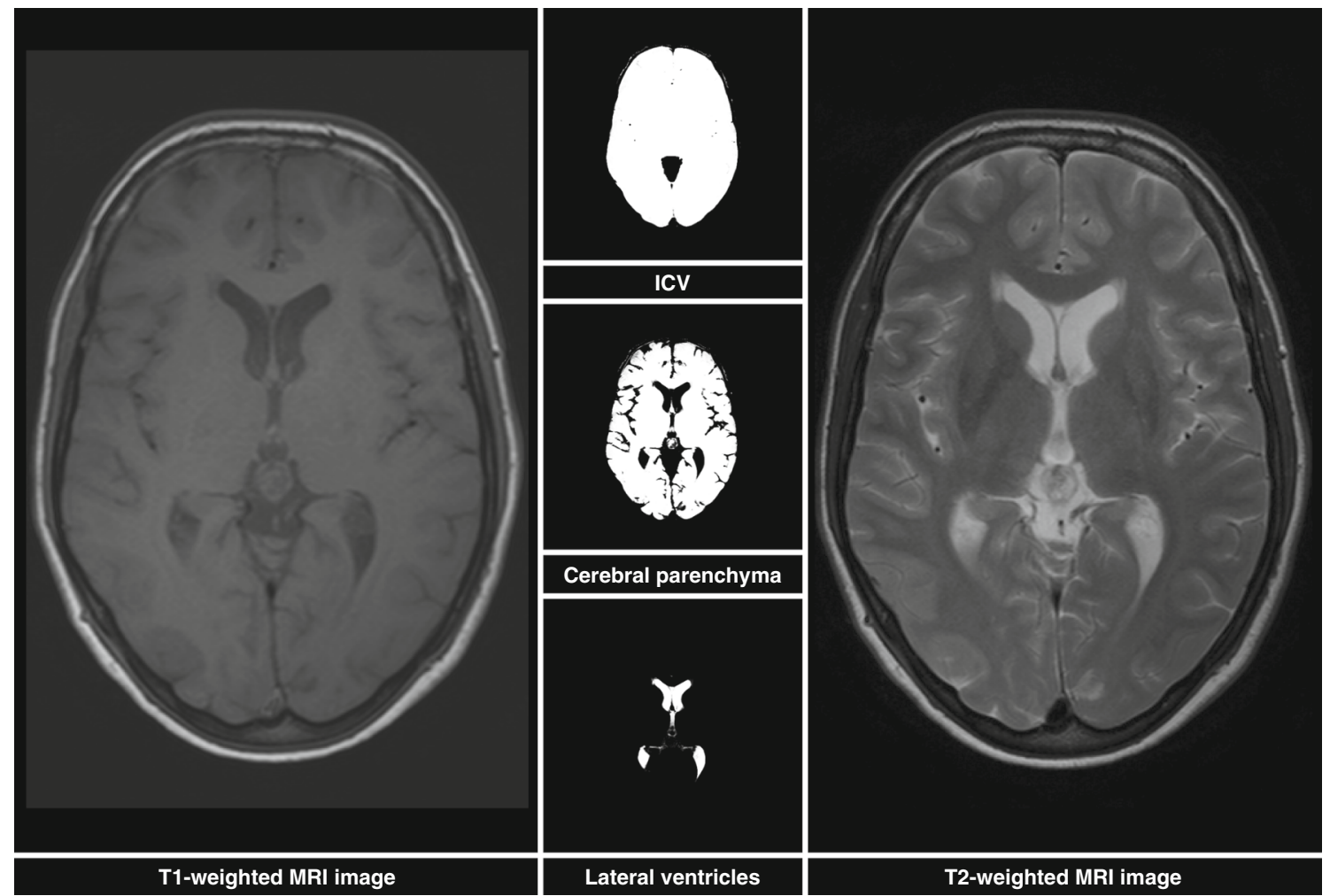

Fig. 1 Example of the segmentations of supratentorial volumes. An example of the T1- and T2-weighted images and the segmentations of supratentorial intracranial volume (ICV), cerebral parenchymal volume, and lateral ventricular volume is shown for a participant

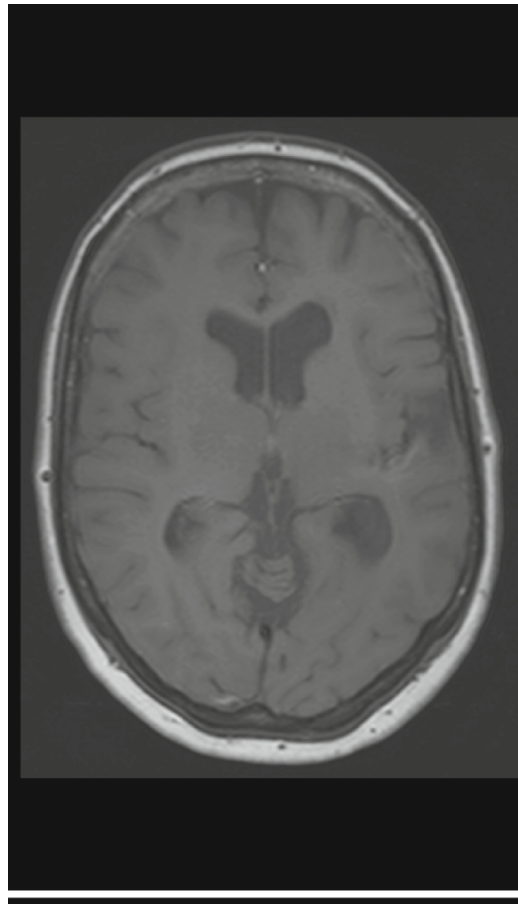

T1-weighted MRI image

Fig. 2 Example of the segmentations in a participant with a cerebral infarct. An example of the T1- and T2-weighted images and the segmentations of supratentorial intracranial volume (ICV), cerebral parenchymal volume, and lateral ventricular volume, and the manual segmentation of infarct volume is shown for a participant with a

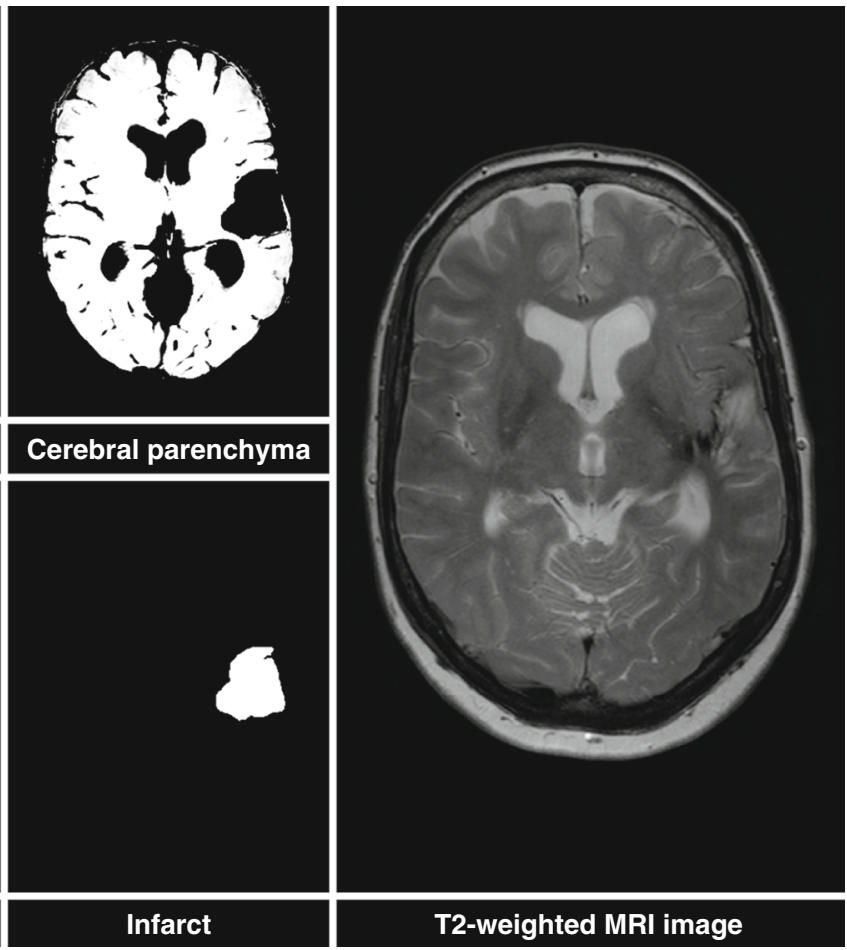

cerebral infarct. As can be seen, small segmentation errors can occur (e.g., due to small flow voids in the ventricles). However, the volume of these segmentation errors is small compared to the absolute volume of the brain structures 
Table 1 Participant characteristics and cerebral volumes

Patients 18 months after aSAH $(n=38)$

$\begin{array}{ll}\text { Characteristics } & \\ \text { Men/women } & 11 / 27 \\ \text { Age at time of MRI (years) } & 53.2 \pm 12.7 \\ \text { Good clinical condition on admission } & 32(84 \%) \\ \text { (WFNS I-III) } & 11(30 \%) \\ \text { Symptomatic hydrocephalus } & 5(15 \%) \\ \text { Delayed cerebral infarction } & 1(3 \%) \\ \text { Rebleeding } & \\ \text { Clinical outcome at time of scan }{ }^{\mathrm{a}} & 8(22 \%) \\ \quad \text { No symptoms (mRS 0) } & 18(48 \%) \\ \quad \text { No disability (mRS 1) } & 8(22 \%) \\ \quad \text { Slight disability (mRS } 2) & 3(8 \%) \\ \quad \text { Moderate disability (mRS } 3) & \\ \text { Cerebral volumes } & \\ \text { Cerebral parenchymal volume }(\mathrm{ml}) & 950 \pm 103 \\ \text { Lateral ventricular volume }(\mathrm{ml}) & 42(21-81) \\ \text { Cerebral infarct volume }(\mathrm{ml})^{\mathrm{c}} & 8.6(1.1-80.9) \\ \text { Presence of cerebral infarcts } & 16(42 \%) \\ \text { Small subcortical }(<1.5 \mathrm{~cm}) & 4(11 \%) \\ \text { Large subcortical }_{\text {Cortical }} & 4(11 \%) \\ \end{array}$

Data are $n$ (percentages), means \pm SD or medians (10-90th percentile)

${ }^{a}$ The mRS from one participant is missing

${ }^{\mathrm{b}}$ Supratentorial volumes

${ }^{\mathrm{c}}$ For cerebral infarct volume, only the volumes of patients who had infarcts are shown

to cerebral parenchymal volume, presence of cerebral infarcts or infarct volumes at 18 months after aSAH. Bicaudate index on admission was related to lateral ventricular enlargement at 18 months (Beta; 95\%CI: 0.51; 0.14↔0.88).

Occurrence of delayed cerebral infarction during hospitalization was related to smaller cerebral parenchymal volumes $(-0.14 ;-0.25 \leftrightarrow-0.04)$, lateral ventricular enlargement $(0.49$; $0.16 \leftrightarrow 0.83)$, and, as expected, also to presence of cerebral infarcts at 18 months after aSAH $(p<0.05)$. Occurrence of symptomatic hydrocephalus during hospitalization was not related to cerebral parenchymal volume, lateral ventricular volume, or infarcts.

Lower cerebral parenchymal volume at 18 months after aSAH was related to a worse clinical outcome $(\mathrm{mRS} \geq 2$ versus a $\mathrm{mRS}<2$ ) (odds ratio; 95\%CI: 91.2; $2.5 \leftrightarrow 3395.1$ ), but lateral ventricular volume was not related to a worse clinical outcome $(1.1 ; 0.2 \leftrightarrow 5.1)$.

\section{Discussion}

A higher bicaudate index at admission after aSAH was related to long-term ventricular enlargement. Also, delayed cerebral infarction was related to long-term ventricular enlargement and to smaller parenchymal volume, whereas measures of the initial impact of the hemorrhage were not related to long-term parenchymal volumes.

We have tuned a method for volume measurements specifically for use on brain MRI scans with a clinical scanning protocol in patients after aSAH [6] and were able to quantify long-term cerebral damage. Our results combined with a previous study showed that different risk factors for poor functional outcome are associated with distinct patterns of cerebral abnormalities after aSAH [4]. The bicaudate index measured at admission was related to long-term ventricular enlargement, but a symptomatic

Table 2 Relationship between known risk factors for poor functional outcome after aSAH and cerebral volumes at 18 months after aSAH $(n=38)$

\begin{tabular}{|c|c|c|c|c|}
\hline & \multicolumn{2}{|c|}{ Cerebral parenchymal volume (ml) } & \multicolumn{2}{|c|}{ Lateral ventricular volume $^{a}$} \\
\hline & $\mathrm{B} ; 95 \% \mathrm{CI}$ & Beta; 95\%CI & $\mathrm{B} ; 95 \% \mathrm{CI}$ & Beta; $95 \% \mathrm{CI}$ \\
\hline \multicolumn{5}{|l|}{ Risk factors determined at admission } \\
\hline Clinical condition on admission (per WFNS grade) & $3.2 ;-5.7 \leftrightarrow 12.1$ & $0.04 ;-0.07 \leftrightarrow 0.14$ & $-0.02 ;-0.19 \leftrightarrow 0.15$ & $-0.04 ;-0.37 \leftrightarrow 0.30$ \\
\hline Amount of extravasated blood (Hijdra score) & $0.38 ;-0.71 \leftrightarrow 1.46$ & $0.04 ;-0.07 \leftrightarrow 0.15$ & $0.01 ;-0.01 \leftrightarrow 0.02$ & $0.11 ;-0.25 \leftrightarrow 0.45$ \\
\hline Bicaudate index & $-1.9 ;-4.7 \leftrightarrow 0.8$ & $-0.09 ;-0.22 \leftrightarrow 0.04$ & $0.06 ; 0.02 \leftrightarrow 0.11 * *$ & $0.51 ; 0.14 \leftrightarrow 0.88^{* *}$ \\
\hline \multicolumn{5}{|l|}{ Risk factors determined during hospitalization } \\
\hline Symptomatic hydrocephalus & $-14 ;-38 \leftrightarrow 10$ & $-0.07 ;-0.18 \leftrightarrow 0.05$ & $0.18 ;-0.29 \leftrightarrow 0.66$ & $0.14 ;-0.23 \leftrightarrow 0.51$ \\
\hline Delayed cerebral infarction & $-38 ;-66 \leftrightarrow-9 *$ & $-0.14 ;-0.25 \leftrightarrow-0.04^{*}$ & $0.80 ; 0.26 \leftrightarrow 1.35^{* *}$ & $0.49 ; 0.16 \leftrightarrow 0.83^{* *}$ \\
\hline
\end{tabular}

The relationships between risk factors for poor functional outcome after aSAH and cerebral parenchymal and lateral ventricular volumes at 18 months after aSAH are shown as regression B coefficients $(95 \% \mathrm{CI})$ and standardized regression beta coefficients $(95 \% \mathrm{CI})$; both adjusted for age, sex, and intracranial volume

${ }^{a}$ Lateral ventricular volumes were natural-log-transformed

${ }^{*} p<0.05 ; * * p<0.01$ 
hydrocephalus during hospitalization was not. Apparently, acute ventricular enlargement is more related to tissue damage than symptomatic hydrocephalus during hospitalization, which is often treated with cerebrospinal fluid drainage. Delayed cerebral infarction during hospitalization proved to be the most important predictor of parenchymal volumes in the long-term.

Occurrence of an aSAH and related complications results in long-term, heterogeneous cerebral damage which, as we have showed, can be quantified. Larger studies are needed to unravel underlying pathophysiological mechanisms. The relation between bicaudate index on admission, but not symptomatic hydrocephalus, and long-term ventricular volumes suggests that effects of early ventricular drainage should also be studied.

A strength of the present study was the prospective design and the systematical analysis of patterns of longterm cerebral abnormalities after aSAH. The used method for cerebral volume measurements has a high accuracy for use on clinical MRI scans [6]. By using this method, we were able to find different patterns of long-term cerebral damage despite the relatively small number of patients. Our method was tuned specifically on brain MRI scans with a clinical scanning protocol and is suitable for post hoc analyses of well-characterized patient populations. With proper tuning, our method can also be used on 3D T1-weighted images.

Limitations of our study could be that our cohort only involved patients who received endovascular treatment after aSAH and who had a relatively favorable outcome. Our method was not tested on patients after surgical treatment. Surgical treatment, however, has additional effects on the studied cerebral volumes [5] that should be taken into account and studied separately from the effect of aSAH alone. Another limitation is that it may be difficult to differentiate between delayed cerebral infarction and new infarcts due to the coiling procedure. Although in none of the patients we had clinical evidence for new infarcts due to coiling and we included as delayed cerebral infarction only patients with clinical symptoms of delayed cerebral infarction and new infarctions compatible with these symptoms, we cannot completely rule out that in some patients the new infarcts were related to aneurysm treatment.

Limitations of volume measurements in general are that severe motion artifacts on MRI and severe MRI artifacts after surgical treatment can hinder accurate volume measurements. Also, small segmentation errors (e.g., due to small flow voids in the ventricles) can still occur due to intersubject differences, and focal cerebral lesions cannot be segmented automatically.

Volume measurements of the brain are able to quantify patterns of long-term cerebral damage in relation to different risk factors after aSAH. Application of volumetric techniques may provide more insight into the heterogeneous underlying pathophysiological processes. After confirmation of these results in larger studies, volumetric measures might even be used as outcome measures in future treatment studies.

Acknowledgments This study was supported financially by a High Potential grant from Utrecht University. JB's research was supported by a Clinical Research Talent Fellowship of the UMC Utrecht.

Ethical standards and patient consent We declare that all human studies have been approved by the UMC Utrecht Ethics Committee and have therefore been performed in accordance with the ethical standards laid down in the 1964 Declaration of Helsinki and its later amendments. We declare that all patients gave informed consent prior to inclusion in this study.

Conflict of interest We declare that we have no conflict of interest.

\section{References}

1. van Gijn J, Kerr RS, Rinkel GJ (2007) Subarachnoid haemorrhage. Lancet 369(9558):306-318

2. Hackett ML, Anderson CS (2000) Health outcomes 1 year after subarachnoid hemorrhage: an international populationbased study. The Australian cooperative research on subarachnoid hemorrhage study group. Neurology 55(5):658-662

3. Wermer MJ, Kool H, Albrecht KW, Rinkel GJ (2007) Subarachnoid hemorrhage treated with clipping: long-term effects on employment, relationships, personality, and mood. Neurosurgery 60(1):91-97, discussion 97-98

4. Bendel P, Koivisto T, Aikia M, Niskanen E, Kononen M, Hanninen T, Vanninen R (2010) Atrophic enlargement of CSF volume after subarachnoid hemorrhage: correlation with neuropsychological outcome. AJNR Am J Neuroradiol 31(2): 370-376

5. Bendel P, Koivisto T, Niskanen E, Kononen M, Aikia M, Hanninen T, Koskenkorva P, Vanninen R (2009) Brain atrophy and neuropsychological outcome after treatment of ruptured anterior cerebral artery aneurysms: a voxel-based morphometric study. Neuroradiology 51(11):711-722

6. de Bresser J, Vincken KL, Kaspers AJ, Rinkel GJE, Viergever MA, Biessels GJ (2012) Quantification of cerebral volumes on MRI 6 months after aneurysmal subarachnoid hemorrhage. Stroke 43(10):2782-2784

7. van Swieten JC, Koudstaal PJ, Visser MC, Schouten HJ, van Gijn J (1988) Interobserver agreement for the assessment of handicap in stroke patients. Stroke 19(5):604-607

8. Schaafsma JD, Velthuis BK, Majoie CB, van den Berg R, Brouwer PA, Barkhof F, Eshghi O, de Kort GA, Lo RT, Witkamp TD, Sprengers ME, van Walderveen MA, Bot JC, Sanchez E, Vandertop WP, van Gijn J, Buskens E, van der Graaf Y, Rinkel GJ (2010) Intracranial aneurysms treated with coil placement: test characteristics of follow-up MR angiography - multicenter study. Radiology 256(1):209-218

9. Anonymous (1988) Report of world federation of neurological surgeons committee on a universal subarachnoid hemorrhage grading scale. J Neurosurg 68(6):985-986

10. van Gijn J, Hijdra A, Wijdicks EF, Vermeulen M, van Crevel H (1985) Acute hydrocephalus after aneurysmal subarachnoid hemorrhage. J Neurosurg 63(3):355-362 
11. Hijdra A, Brouwers PJ, Vermeulen M, van Gijn J (1990) Grading the amount of blood on computed tomograms after subarachnoid hemorrhage. Stroke 21(8):1156-1161

12. Vergouwen MD, Vermeulen M, van Gijn J, Rinkel GJ, Wijdicks EF, Muizelaar JP, Mendelow AD, Juvela S, Yonas H, Terbrugge KG, Macdonald RL, Diringer MN, Broderick JP, Dreier JP, Roos YB (2010) Definition of delayed cerebral ischemia after aneurysmal subarachnoid hemorrhage as an outcome event in clinical trials and observational studies: proposal of a multidisciplinary research group. Stroke 41(10):2391-2395

13. de Bresser J, Portegies MP, Leemans A, Biessels GJ, Kappelle LJ, Viergever MA (2011) A comparison of MR based segmentation methods for measuring brain atrophy progression. Neuroimage 54(2):760-768 